ARTICLE

https://doi.org/10.1038/s41467-020-20409-4 OPEN

\title{
Slowing down DNA translocation through solid- state nanopores by edge-field leakage
}

\author{
Ceming Wang (1) ${ }^{1}$, Sebastian Sensale ${ }^{2}$, Zehao Pan ${ }^{1}$, Satyajyoti Senapati (i) ${ }^{1}$ \& Hsueh-Chia Chang (id ${ }^{1,2}{ }^{凶}$
}

Solid-state nanopores allow high-throughput single-molecule detection but identifying and even registering all translocating small molecules remain key challenges due to their high translocation speeds. We show here the same electric field that drives the molecules into the pore can be redirected to selectively pin and delay their transport. A thin high-permittivity dielectric coating on bullet-shaped polymer nanopores permits electric field leakage at the pore tip to produce a voltage-dependent surface field on the entry side that can reversibly edge-pin molecules. This mechanism renders molecular entry an activated process with sensitive exponential dependence on the bias voltage and molecular rigidity. This sensitivity allows us to selectively prolong the translocation time of short single-stranded DNA molecules by up to 5 orders of magnitude, to as long as minutes, allowing discrimination against their double-stranded duplexes with $97 \%$ confidence.

\footnotetext{
${ }^{1}$ Department of Chemical and Biomolecular Engineering, University of Notre Dame, Notre Dame, IN, USA. ${ }^{2}$ Department of Aerospace and Mechanical Engineering, University of Notre Dame, Notre Dame, IN, USA. ${ }^{\bowtie}$ email: hchang@nd.edu
} 
S olid-state and protein nanopores are an emerging class of single-molecule sensors for DNA sequencing ${ }^{1-3}$, protein detection $^{1,4,5}$, and DNA-protein complex analyses ${ }^{6}$. The Achilles heel of nanopores has been the inability to control the motion of biomolecules during voltage-driven translocation through the pore ${ }^{1,3,7-9}$. With the development of enzyme-based methods that ratchet polynucleotides through the pore, the first nanopore-sequencer has been realized using protein nanopores ${ }^{3}$. Despite the progress that has been made with biological nanopores, solid-state nanopores with high stability and tunable pore diameters still offer several advantages. They facilitate integration with compact electronic/optical sensor modalities and allow higher throughput than their protein counterparts. However, developing solid-state nanopore sensors capable of complete characterization of the translocating biomolecules has been challenging ${ }^{1,7,9}$, primarily because of the fast electrophoretic translocation by highly focused electric fields at the pore. The high fields are due to the nanoscale pore dimensions necessary for resistive current signals above thermal noise, and the minimum bias voltage $(20-60 \mathrm{mV})^{10}$ necessary to overcome barriers due to conformation entropy, electrostatic repulsion, and electro-osmotic flow ${ }^{11,12}$.

Typical electrophoretic velocities of nucleic acids across solidstate nanopores are $10-1000 \mathrm{~ns}$ per base ${ }^{1}$. At these high velocities, short nucleic acids $(<100 \mathrm{nt})$ as well as small protein molecules are often undetected, much less identified ${ }^{13}$. Thus, a high signal bandwidth $(>1 \mathrm{MHz})$ is needed to fully resolve the resistive pulses $^{14,15}$. High signal bandwidth, however, also strongly amplifies thermal noise in the current recordings; this noise makes the signal resistive pulses become undetectable ${ }^{16}$. This limitation hence prevents accurate profiling of promising cancer biomarkers like proteins, short mRNA fragments, and microRNAs (19-22 nt) by solid-state nanopores ${ }^{13,17,18}$. For the proposed sequencing applications by quantum tunneling, speed control is also a key issue for realizing practical quantum sequencers ${ }^{9}$. An additional mechanism to dramatically reduce (by orders of magnitude) and control the fast electrophoretic velocity of molecules is therefore necessary to realize sensitive and selective solid-state nanopore sensors for short nucleic acids, and other small biomolecules ${ }^{13}$ and sequencing platforms ${ }^{2,19}$.

Multiple approaches have been proposed to slow down the translocation events ${ }^{20}$, which involve either modifying the properties (mostly viscosity) of the electrolyte ${ }^{10,21,22}$, incorporating optical (or magnetic) traps or tweezers ${ }^{20,23,24}$, or using protein tags to slow down the motion of the smaller molecules ${ }^{25-27}$. In the last few years, surface charge density modulation has also been suggested to slow down translocation events $^{28-33}$, mostly by building nanopores with dielectric materials like $\mathrm{Al}_{2} \mathrm{O}_{3}{ }^{29,32,34,35}$ and $\mathrm{HfO}_{2}{ }^{30}$, or by exploring optoelectronic control of surface charge ${ }^{33}$. However, these modifications produce a gating field that is much weaker than the driving field along the pore and are only capable of reducing the translocation speed by at most one order of magnitude $29,30,33,35$, which is small compared to the typical two-decade wide bandwidth of the Poisson distribution of translocation times. Interference with the sensing current signals is also a problem ${ }^{9}$. An intriguing approach has been reported that uses the leakage electric fields to manipulate and preconcentrate DNA in nanofluidic channels ${ }^{36}$. Since the electric field can leak through high-permittivity materials, the leakage field enters the solid surface as an intense normal field that can arrest the transport of biomolecules and trap them at the surface.

In this article we will show that the deposition of a highpermittivity $\mathrm{Al}_{2} \mathrm{O}_{3}$ film over an insulating bullet-shaped polymer nanopore allows the field to leak through the dielectric material and into the upper membrane, producing a field comparable in intensity to the translocation field yet sufficiently weak to prevent permanent trapping of the molecules (and clogging of the nanopores). By properly tuning the bullet-shaped geometry, which has a conical base and a short straight pore at the tip, this field can induce a net voltage-dependent surface charge density on the upper membrane, which can reversibly edge-pin flexible translocating molecules, rendering molecular entry into the pore an activated process. The translocation time becomes a strong function of the molecular rigidity, which is ideally suited for discriminating between short $(<100 \mathrm{nt})$ single-stranded and duplex nucleic acids whose persistence length differ by 2 orders of magnitude. We can selectively prolong the translocation time of short single-stranded DNA molecules by 5 orders of magnitude, thus allowing discrimination against their double-stranded duplexes with $97 \%$ confidence. Since the leakage field is outside the nanopore, it does not interfere with the resistive signal current from within the pore tip.

\section{Results}

Electric field leakage through dielectric materials. Ideal dielectrics are assumed to be perfect insulators ${ }^{37}$ (that is, they present infinite resistivity). However, in reality, their resistivity is finite, leading to a passage of current when subjected to applied voltages commonly known as current leakage ${ }^{37,38}$. This leakage is often undesirable, as it decreases the effective electric field needed for the functioning of multiple devices and promotes material degradation processes ${ }^{38-40}$. In materials often used in nanopores, such as $\mathrm{SiO}_{2}, \mathrm{Si}_{3} \mathrm{~N}_{4}$ and $\mathrm{Al}_{2} \mathrm{O}_{3}$, this leakage is often associated with Poole-Frenkel effects ${ }^{37,41}$ and it manifests at voltages ${ }^{42}$ of the order of $10^{9} \mathrm{~V} \mathrm{~m}^{-1}$. There is no significant current leakage at lower electric fields. However, due to the finite permittivity of these materials, field lines can penetrate the dielectric film leading to significant field leakage ${ }^{43,44}$.

Optical and electric intensity can become singular at metallic or dielectric cones or wedges. These singular fields are present in tip plasmonics ${ }^{45}$, knife-edge scattering 46 , Taylor cones of electrified drops ${ }^{47}$, etc. In our earlier microchannel electro-osmosis work, the singular tangential electric field at a 90-degree turn of an insulating wall was converted into a comparably singular leakage field across the corner by introducing finite wall permittivity 43,44 . The leakage field exits the other side of the corner as an intense normal field that can arrest the transport of micro-colloids and trap them at the upstream side of the corner. This same mechanism can be incorporated into nanopore devices, leading to high electric fields normal to the upper membrane of the nanopore which are capable to pin the molecules to the tip of the nanopore.

Our designed solid-state nanopore has a conical base and a short straight pore at the tip, which is coated with a highly conformal $\mathrm{Al}_{2} \mathrm{O}_{3}$ film (see Fig. 1a and Supplementary Fig. 1). The conical base with the insulating PET (polyethylene terephthalate) membrane wall focuses the electric field and the high-permittivity $\mathrm{Al}_{2} \mathrm{O}_{3}$ film on the straight pore edge facilitates field leakage at the tip end (see Fig. 1b). Once the field lines enter the dielectric film inside the pore, the axially conditioned parallel field lines within the straight pore region ensure that the field intensity in the dielectric film is identical to that in the aqueous phase in the pore, despite the higher permittivity of the latter phase. With the converging geometry at the conical base, the field lines are confined to the aqueous bulk. A simple Gauss volume flux balance then allows us to relate the normal leakage field $E_{\text {leak }}$ and the average electric field $E_{0}$ in the pore entrance at the neck with the conic base,

$$
E_{\text {leak }}=E_{\text {film }}^{\text {normal }}\left(\frac{\varepsilon_{\text {film }}}{\varepsilon_{\text {water }}}\right)=\frac{E_{0} \varepsilon_{\text {film }}}{\varepsilon_{\text {water }}+\varepsilon_{\text {film }}\left((1+l / R)^{2}-1\right)},
$$

where $\varepsilon_{\text {water }}$ and $\varepsilon_{\text {film }}$ are the permittivity of water and dielectric film, respectively, $R$ is the radius of nanopore orifice, and $l$ is the 
a

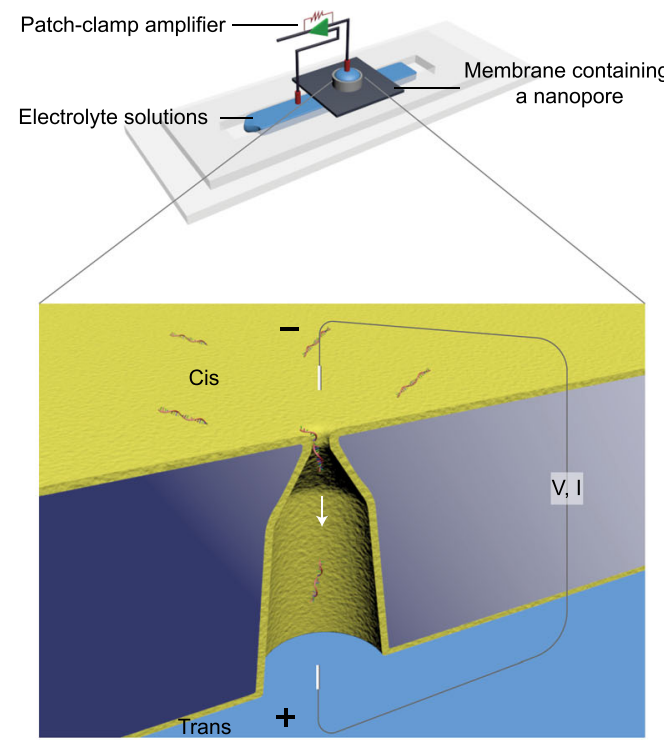

b

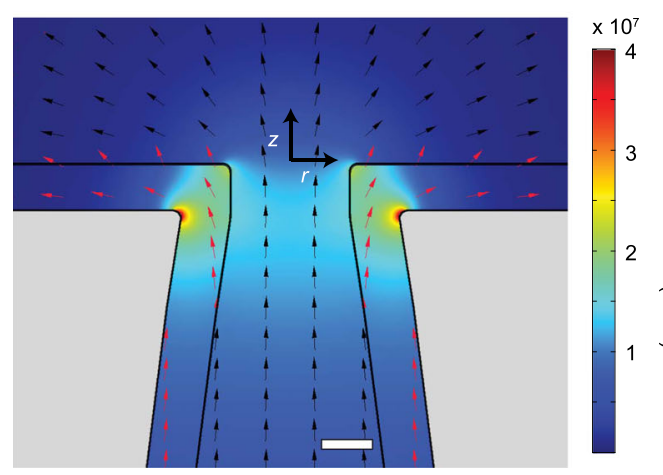

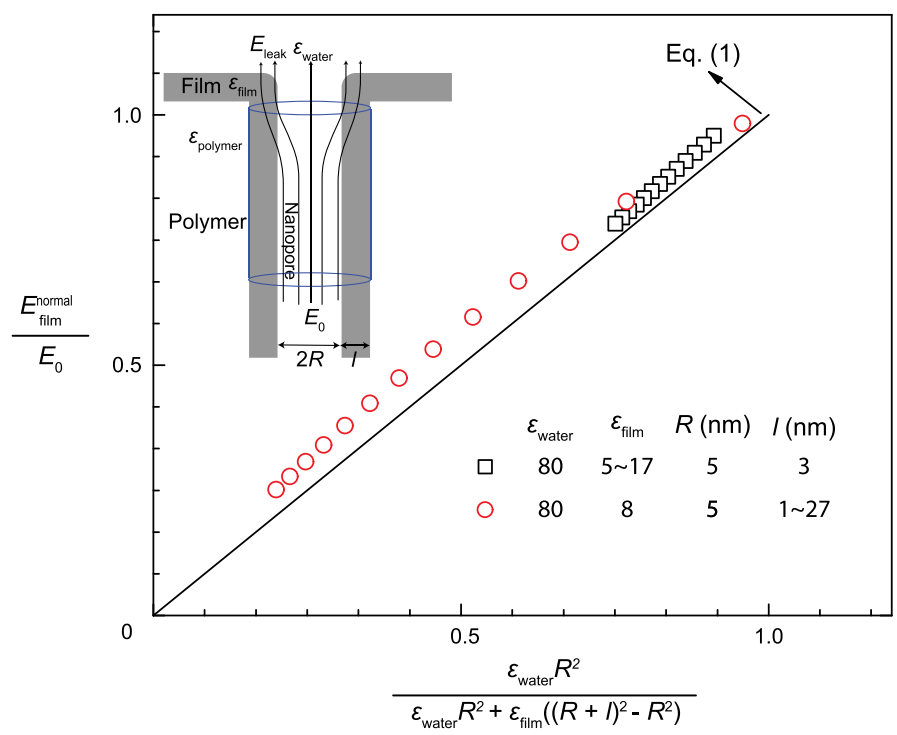

d

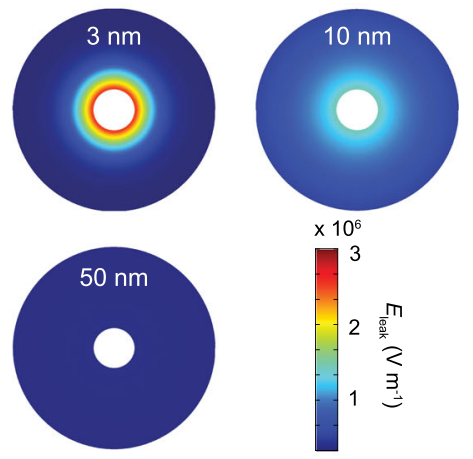

Fig. 1 The electric field leakage effect in $\mathbf{A l}_{2} \mathbf{O}_{\mathbf{3}}$-coated polymer nanopores. a Set-up to measure resistive pulses from the translocation of individual DNA molecules through single bullet-shaped polymer nanopore coated with a thin $\mathrm{Al}_{2} \mathrm{O}_{3}$ layer. $\mathbf{b}$ Electrostatic modeling of an $\mathrm{Al}_{2} \mathrm{O}_{3}$-coated bullet-like nanopore (tip diameter: $8 \mathrm{~nm}$, half cone angle: $8^{\circ}$ ) simulated with an applied voltage of $500 \mathrm{mV}$. Electric field direction and intensity were evaluated numerically on the tip side of the $\mathrm{Al}_{2} \mathrm{O}_{3}$-coated polymer nanopore. The electric field is significantly enhanced and develops a normal field leakage near the sharp pore edge. Scale bar $=3 \mathrm{~nm}$. c Validation of Eq. (1) through the use of finite-element-method simulations for normal field leakage in the dielectric film. Different permittivities (black squares, $\varepsilon_{\text {film: }}$ : 17 ) and film thicknesses (red circles, l: 1 27 nm) were sampled. Inset shows the schematics of a high-permittivity dielectric film on an insulating polymer nanopore orifice and the Gauss volume used to estimate the leakage field around the pore. $\mathbf{d}$ Left: Surface plots of the strength of normal leakage field $\left(E_{\text {leak }}\right.$ ) showing the normal leakage field at the pore edge is a strong function of $\mathrm{Al}_{2} \mathrm{O}_{3}$ film thickness (nanopore diameter, $8 \mathrm{~nm}$ ). Right: Axial dependence of the normal leakage field as a function of distance from the pore mouth $r$ for three $\mathrm{Al}_{2} \mathrm{O}_{3}$ film thicknesses.

thickness of the dielectric film (see Fig. 1c). $E_{\text {film }}^{\text {normal }}$ is the field inside the dielectric membrane, which can be considered to be equal to the field in the liquid region $E_{\text {water }}^{\text {normal }}$ (near the tip of the pore) for large pores and small values of $l$ (see Supplementary Note 1). Two limits of Eq. (1) are instructive. For $l / R$ approaching infinity, corresponding to a non-polymeric dielectric membrane whose area is much larger than the pore tip area, $E_{\text {leak }} / E_{0}$ scales as $(R / l)^{2} \ll 1$. This indicates conventional solid-state nanopores fabricated in dielectric membranes (such as $\mathrm{SiN}, \mathrm{SiO}_{2}$, and $\mathrm{Al}_{2} \mathrm{O}_{3}$, etc.) cannot produce significant molecule-pinning field at the pore edge, as the field is distributed over a large surface area that scales as $R^{2}$. Indeed, to date, there has been no experimental report of prolonging translocation times in dielectric membrane nanopores by edge-field leakage. In fact, this field penetration across the entire dielectric membrane causes significant dielectric noise in the nanopores ${ }^{14,48,49}$. The other limit of $l / R$ approaching zero yields that $E_{\text {leak }} \sim E_{0}\left(\varepsilon_{\text {film }} / \varepsilon_{\text {water }}\right)$. The leakage field would then be proportional to the applied field and of comparable intensity if the permittivity ratio is not too small. Hence, a compound pore, with a nearly insulating polymer membrane and a thin high-permittivity dielectric film, is necessary for a leakage field with intensity and dimension that can delay the molecular translocation time without generating significant noise in the resistive signal. Finite-element-method (FEM) simulations confirm that the electric field intensity within the dielectric film increases considerably and develops a normal field leakage when approaching the pore edge. In Fig. 1d, the intensity of normal leakage field along the $r$ axis is shown for different film thicknesses $l$ $(3-50 \mathrm{~nm})$. The rapidly increasing (singular) intensity of normal field at the pore edge with decreasing $l$ confirms enhanced field leakage in PET nanopores coated with a thin $\mathrm{Al}_{2} \mathrm{O}_{3}$ film. Note that the zero-thickness limit is singular, as the field at the pore edge would be purely tangential for a perfectly insulating membrane.

To create our nanopores, we fabricate single nanopores with asymmetric shapes in PET membranes by the track-etching method ${ }^{50}$ and subsequently deposit an $\mathrm{Al}_{2} \mathrm{O}_{3}$ dielectric film on the pore wall by atomic layer deposition (ALD) ${ }^{51}$. The strength of the electric field at the pore tip can be fine-tuned through the choice of cone angles under the same applied voltage. The as- 


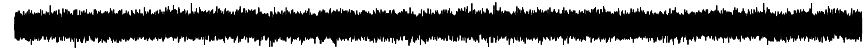

Bullet-shaped nanopore with $3 \mathrm{~nm} \mathrm{Al}_{2} \mathrm{O}_{3}$ film

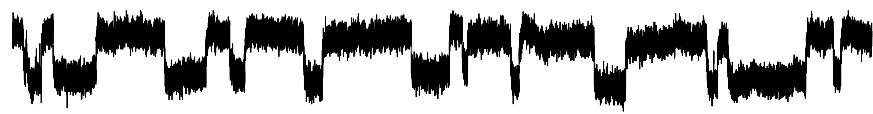

Bullet-shaped nanopore with $10 \mathrm{~nm} \mathrm{Al}_{2} \mathrm{O}_{3}$ film
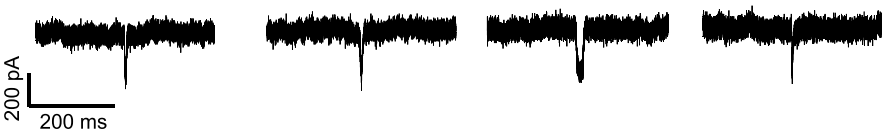
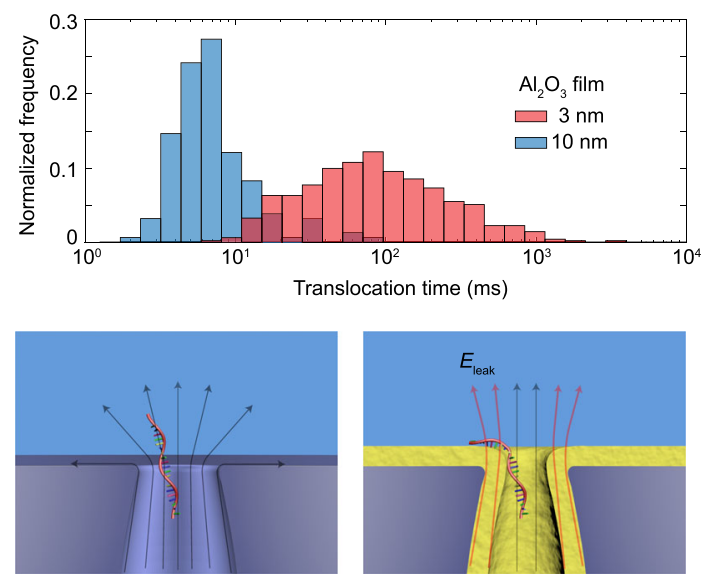

Fig. 2 Slowing down ssDNA translocation speed with electric field leakage. a Representative current traces for 22 nt ssDNA translocation through a bare bullet-shaped PET nanopore without $\mathrm{Al}_{2} \mathrm{O}_{3}$ film coating (diameter, $16 \mathrm{~nm}$ ) and two bullet-shaped nanopores coated with $3 \mathrm{~nm}$ or $10 \mathrm{~nm} \mathrm{Al}_{2} \mathrm{O}_{3}$ film under an applied voltage of $500 \mathrm{mV}$. Both $\mathrm{Al}_{2} \mathrm{O}_{3}$-coated nanopores have the same final tip diameter (10 nm). All three nanopores have similar bullet-like shapes (half cone angle, $7 \pm 2^{\circ}$ ). Slow translocation of 22 nt ssDNA is observed using nanopores with thin $\mathrm{Al}_{2} \mathrm{O}_{3}$ film coating and the average translocation time is a function of film thickness. b Normalized histogram of translocation times for nanopores with $3 \mathrm{~nm}$ or $10 \mathrm{~nm} \mathrm{Al}_{2} \mathrm{O}_{3}$ film. $\mathrm{Average}$ translocation time: $3 \mathrm{~nm}$ $\mathrm{Al}_{2} \mathrm{O}_{3}$ film, $159 \mathrm{~ms}$ ( $n=492$ events); $10 \mathrm{~nm}, 13 \mathrm{~ms}$ ( $n=46$ events). c Schematic showing the dominant tangential electric field at the bare PET nanopore edge results in a fast translocation of $22 \mathrm{nt}$ ssDNA (left) while the normal leakage field at the $\mathrm{Al}_{2} \mathrm{O}_{3}$-coated nanopore edge traps the ssDNA and thus reduces its mobility.

a

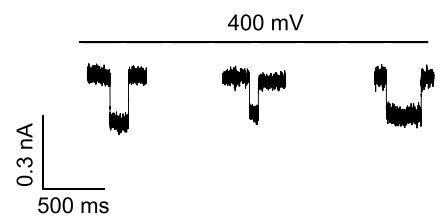

b

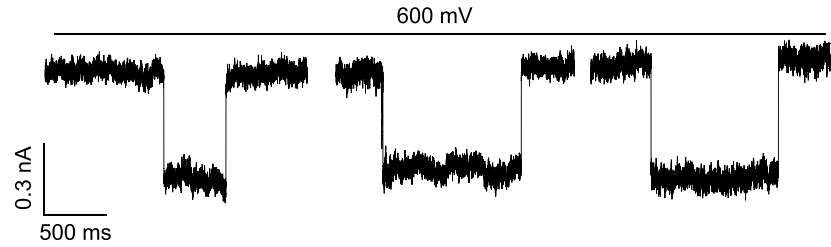

c

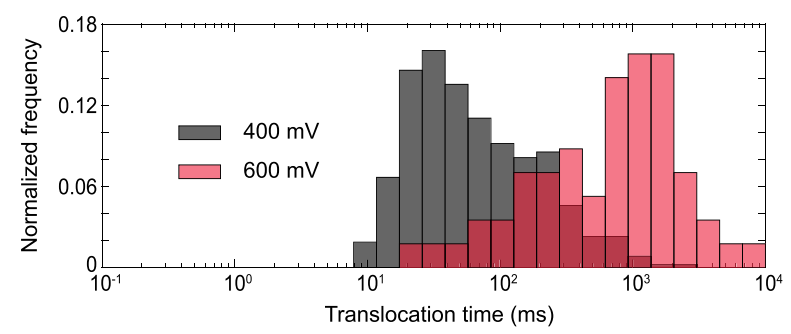

Fig. 3 Effects of electric field leakage on ssDNA transport. a

Representative translocation signals for 22 nt ssDNA translocations at applied voltages of $400 \mathrm{mV}$. b Representative translocation signals for 22 nt ssDNA translocations at applied voltages of $600 \mathrm{mV}$. c Normalized histogram of corresponding translocation times at applied voltages of 400 $\mathrm{mV}(n=480$ events) and $600 \mathrm{mV}(n=57$ events $)$ and average

translocation time as a function of applied voltage. Increasing the strength of electric field leakage can increase the translocation time of ssDNA. Data were acquired using a nanopore coated with $3 \mathrm{~nm} \mathrm{Al}_{2} \mathrm{O}_{3}$ film (diameter, 8 $\mathrm{nm}$; half cone angle, $9 \pm 2^{\circ}$ ). fabricated nanopores with large cone angles have a bullet-like shape while nanopores with small cone angles have a trumpet-like shape $^{52}$ (Supplementary Fig. 1). PET is highly insulating and has been used as supporting substrate to significantly reduce the dielectric noise of nanopores in dielectric membranes ${ }^{49} . \mathrm{Al}_{2} \mathrm{O}_{3}$ has a large dielectric constant of 8 . ALD offers precise control of nanoscale film thickness.

Field leakage induced delay of DNA translocation. Having fabricated the $\mathrm{Al}_{2} \mathrm{O}_{3}$-coated PET nanopores, we next tested the field leakage effect on the translocation of DNA molecules. We selected 22 nucleotides (nt) long single stranded DNA (ssDNA) molecules as representative small nucleic acid molecules whose fast translocation poses a major challenge to their detection by other solid-state nanopores ${ }^{17}$. Figure $2 \mathrm{a}$ presents typical current traces recorded during the translocation of these molecules for a bare bullet-shaped PET nanopore without $\mathrm{Al}_{2} \mathrm{O}_{3}$ film coating and for one with a $3 \mathrm{~nm} \mathrm{Al} \mathrm{Al}_{2} \mathrm{O}_{3}$ film coating (diameter $10 \mathrm{~nm}$ ). Resolvable signals due to translocation events are only observed in bullet-shaped nanopores coated with $\mathrm{Al}_{2} \mathrm{O}_{3}$ films, where substantial field leakage occurs. Moreover, the translocation time strongly depends on the $\mathrm{Al}_{2} \mathrm{O}_{3}$ film thickness. By comparing these two nanopores, we see an increase of one order of magnitude in the observed average translocation time, from 13 to $159 \mathrm{~ms}$ (see Fig. 2b), when the thickness of $\mathrm{Al}_{2} \mathrm{O}_{3}$ film decreases from $10 \mathrm{~nm}$ to $3 \mathrm{~nm}$. As suggested from our field flux balance, the field leakage at the pore edge is indeed expected to become stronger with thinner $\mathrm{Al}_{2} \mathrm{O}_{3}$ dielectric layer. In contrast, no translocation event is detected for bare bullet PET nanopores without the highpermittivity dielectric layer that sustains field leakage-it is a singular limit. Due to limitations on the detection electronics, translocation of $22 \mathrm{nt}$ ssDNA through the bare bullet PET nanopore is too fast to be detected. We verify this by translocating lambda DNA (48.5 kbp) through the bare pore. We found unique blockage signatures not observed for $22 \mathrm{nt}$ ssDNA. Importantly, the average translocation time for lambda DNA was $2.6 \mathrm{~ms}$, translating into $54 \mathrm{~ns}$ per base or $1.2 \mu$ s for $22 \mathrm{nt}$ ssDNA, which is 

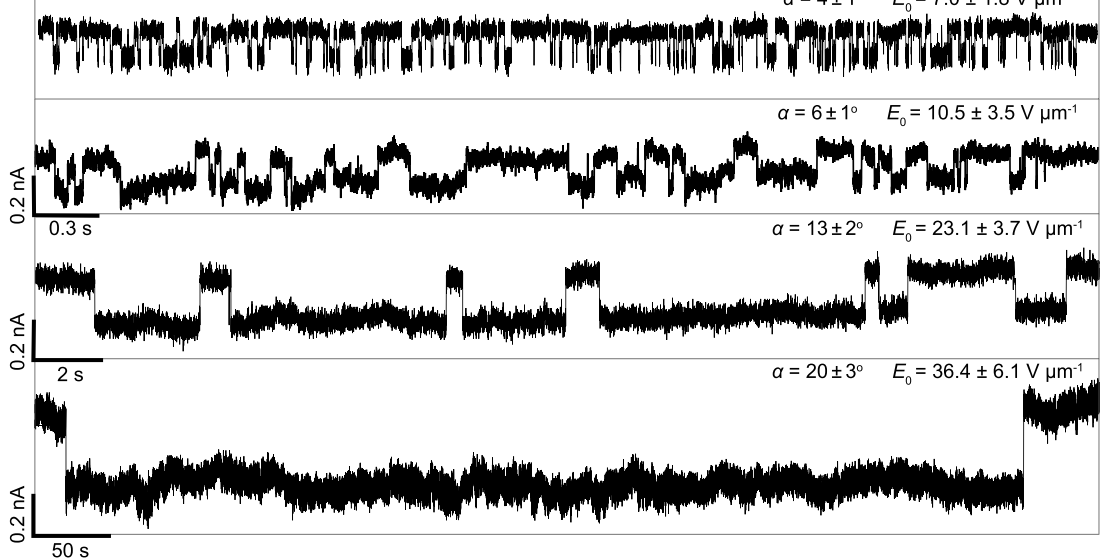

b
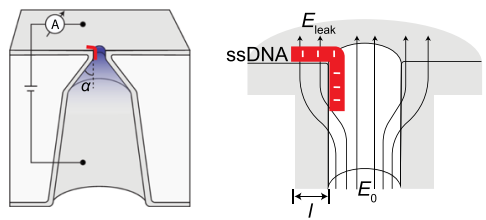

c

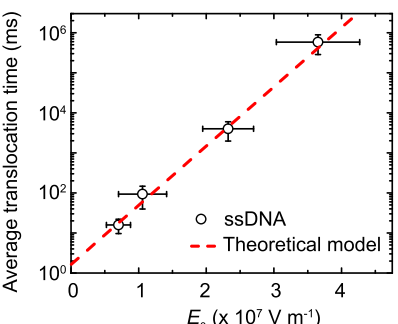

Fig. 4 Modulation of translocation dynamics by angle control. a Representative current traces of 22 nt ssDNA translocating through four bullet-shaped $\mathrm{Al}_{2} \mathrm{O}_{3}$-coated (thickness, $3 \mathrm{~nm}$ ) nanopores (diameter, $10 \mathrm{~nm}$ ) with different half cone angles $(\alpha)$. The nanopores with larger cone angle allow more electric field ( $E_{0}$, as indicated) to be focused at the nanopore tip under the same applied voltage ( $500 \mathrm{mV}$ ) and thus higher magnitude of normal leakage field at the pore edge. With the increase of half cone angle and thus normal leakage field, the average translocation time can be increased exponentially from milliseconds to hundreds of seconds. $\mathbf{b}$ Left: schematic of the measurement apparatus using a bullet-shaped $\mathrm{Al}_{2} \mathrm{O}_{3}$-coated nanopores with half cone angles of $\alpha$. Right: zoom in of the nanopore orifice with ssDNA electrostatically trapped at the pore edge by the normal leakage field. c The average translocation time dependence of $E_{0}(n=3)$. Error bars in the figure represent the standard deviation between independent experiments. The line represents the fit of the data to the theoretical model $\tau=\tau_{0} e^{-\left(\Delta W+\Delta W^{*}\right) / k_{B} T}$, where $\Delta W$ and $\Delta W^{\star}$ are given by Eqs. (3) and (4), respectively, with $\tau_{0}=1.595 \mathrm{~ms}$, a charge per nt of $0.1 e$, and a length per nucleotide of $0.64 \mathrm{~nm}^{62}$.

undetectable (see Supplementary Fig. 2). The correlation between dielectric film thickness and translocation time is observed in all tested $\mathrm{Al}_{2} \mathrm{O}_{3}$-coated PET nanopores at different bias voltages.

Other than dielectric film thickness, different field leakage strengths can also be fine-tuned by varying cone angles of nanopore or bias voltages, as expected from Eq. (1). Previous studies have shown that the translocation of $100 \mathrm{nt}$ ssDNA can be slowed down to $\sim 0.18 \mathrm{~ms}$ using solid-state nanopores in $\mathrm{Al}_{2} \mathrm{O}_{3}$ membranes ${ }^{29}$. We observed an average translocation time $\sim 125 \mathrm{~ms}$ for $22 \mathrm{nt}$ ssDNA in $\mathrm{Al}_{2} \mathrm{O}_{3}$-coated PET nanopores (half cone angle $\sim 9 \pm 2^{\circ}$, diameter $8 \mathrm{~nm}$, applied voltage 400 $\mathrm{mV}$ ). Due to confinement effects and electrostatic interactions, translocation times of DNA molecules (and other small molecules) through small-diameter (and/or charged) nanopores are well-modeled as activated processes ${ }^{53-56}$ $\tau=\frac{h}{k_{\mathrm{B}} T} e^{\Delta G / k_{\mathrm{B}} T}$, where $\tau$ is the translocation time, $\Delta G$ is the height of the activation barrier, $k_{\mathrm{B}}$ is Boltzmann constant, $h$ is the Planck constant, and $T$ is the temperature of the system ${ }^{53-55}$. As the electric field $E_{0}$ pulls the stalled DNA into the pore with a force $q E_{0}$, where $q$ is the effective charge of the molecule, translocation times decrease exponentially with the field in such activated entries, reducing the barrier by $W \sim \int_{0}^{L} q E_{0} d z$ associated to the work done by the applied field to move the DNA molecule a distance $L^{54,55}$. Translocation times can then be written through $\tau=\tau_{0} e^{-W / k_{\mathrm{B}} T}$, where $\tau_{0}$ is the zero-field translocation time, $k_{\mathrm{B}}$ is Boltzmann constant, and $T$ is the temperature of the system ${ }^{53-55}$. In contrast, larger diameter nanopores with small surface charge densities only weakly interact with the translocating molecule and thus they do not exhibit activation barriers, leading to translocation times inversely proportional to the external electric fields, as the electrostatic forces balance with hydrodynamic drag ${ }^{57-59}$. For our $\mathrm{Al}_{2} \mathrm{O}_{3}$-coated PET nanopores, when the applied voltage was slightly increased from 400 to $600 \mathrm{mV}$, the average ssDNA translocation time increased five-fold, from 125 to $1217 \mathrm{~ms}$ (see Fig. 3), suggesting that field leakage increases the activation barrier for ssDNA, opposing translocation as illustrated in Fig. 2c.
To realize the full potential of field leakage induced retardation of ssDNA, we investigated different geometries to enhance field leakage. We use a series of bullet $\mathrm{Al}_{2} \mathrm{O}_{3}$-coated nanopores (diameter, $10 \mathrm{~nm}$ ) with different half cone angles at their conical base. Nanopores with larger half cone angles $\alpha$ allow more electric field to be focused at the nanopore tip under the same applied voltage, as ${ }^{51}$

$$
E_{0} \sim \frac{V}{L^{\prime}}+\frac{V \operatorname{tg} \alpha}{R},
$$

with $L^{\prime}$ the length of the nanopore (11.5 $\mu \mathrm{m}$ in all our experiments). Therefore, larger half cone angles allow higher magnitude of normal field leakage, according to Eq. (1). Such asymmetric nanopores with different half cone angles were fabricated by varying etching times after breakthrough (see Supplementary Fig. 3). Figure 4a compares representative current traces through four $\mathrm{Al}_{2} \mathrm{O}_{3}$-coated nanopores with half cone angles ranging from $4^{\circ}$ to $20^{\circ}$. The magnitude of corresponding electric field $E_{0}$ at the pore tip is indicated in Fig. $4 \mathrm{a}$, while translocation times are presented in Fig. 4c. Strikingly, with the increase of $E_{0}$ and thus normal leakage field, the average translocation time can be increased exponentially from tens of milliseconds to hundreds of seconds due to the activated nature of the entry.

The high tunability of molecular pinning mechanism by varying the leakage field allows versatile control of translocation processes, which is difficult for other interactions ${ }^{29}$. It is expected that the charge, length, and mechanical properties of the translocating molecules can sensitively change the barrier and the translocation time, since the normal leakage field is confined to a film less than 3 $\mathrm{nm}$ in width. To test this selectivity, we analyzed translocation events of 22 base-pairs (bp) long double stranded DNA (dsDNA) molecules. The sample current traces for typical dsDNA translocation events and translocation time histograms at applied voltages of 400 and $600 \mathrm{mV}$ are presented in Fig. 5. Interestingly, at both voltages, the translocation speed for dsDNA is observed to be orders of magnitude faster than that for the ssDNA. For example, at 400 $\mathrm{mV}$, the average translocation time of dsDNA is around $4 \mathrm{~ms}$, which is 2 orders of magnitude shorter than that of the ssDNA (and 2 orders of magnitude larger than reported translocation times in 
a

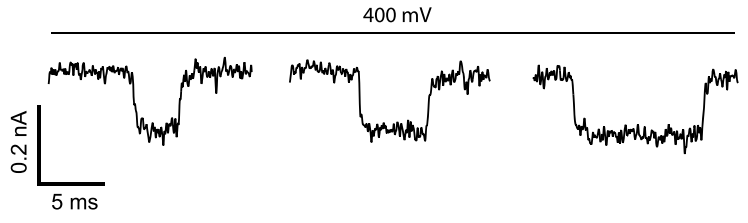

b

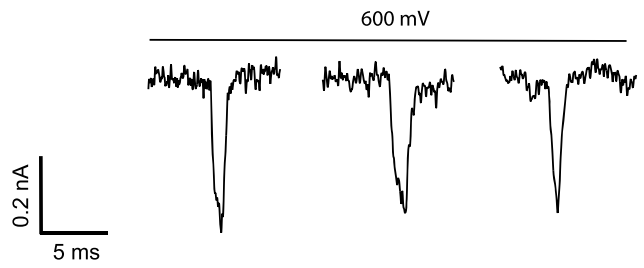

c

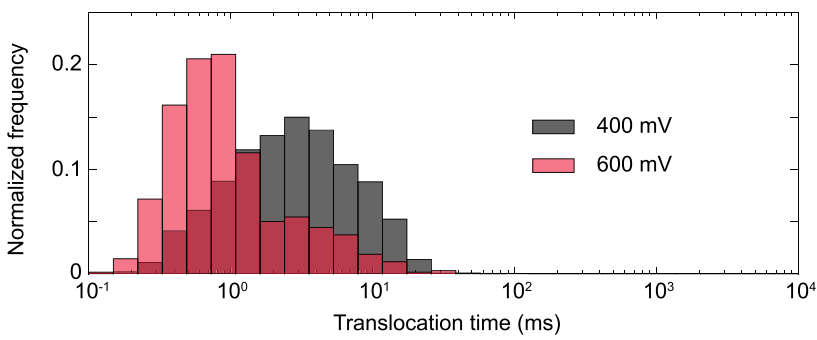

Fig. 5 Effects of electric field leakage on dsDNA transport. a

Representative translocation signals for 22 bp dsDNA translocations at applied voltages of $400 \mathrm{mV}$. b Representative translocation signals for 22 bp dsDNA translocations at applied voltages of $600 \mathrm{mV}$. c Normalized histogram of corresponding translocation times at applied voltages of 400 $\mathrm{mV}(n=701$ events) and $600 \mathrm{mV}(n=1762$ events $)$ and average translocation time as a function of applied voltage. Increasing the strength of electric field leakage decreases the translocation time of dsDNA. Data were acquired using a nanopore coated with $3 \mathrm{~nm} \mathrm{Al} \mathrm{O}_{3}$ film (diameter, 8 $\mathrm{nm}$; half cone angle, $9 \pm 2^{\circ}$ ).

other nanopores ${ }^{17,29}$, see Supplementary Fig. 4). The translocation time of dsDNA has an opposite voltage-dependence to the ssDNA. Increasing the applied voltage from 400 to $600 \mathrm{mV}$, the average translocation time of dsDNA decreases from 4 to $1.5 \mathrm{~ms}$, suggesting that the normal leakage field has much less effect on dsDNA translocation than on its ssDNA counterpart. With the opposite trends of ssDNA and dsDNA translocation times on voltage bias, the mean translocation times of the two molecules are about a factor of $~ 811$ different at $600 \mathrm{mV}$ and, taking into account the spread in their distributions, the probability of a ssDNA exhibiting the same translocation time as a dsDNA is less than 3\% (Fig. 6a). Since an excess of ssDNA molecules with long translocation times will increase the assay time for a given number of translocation events, the selectivity gained at high field comes with a trade-off in longer assay time for ssDNA-rich mixtures (see Supplementary Fig. 5).

To explain these differences in translocation times, we may estimate the influence of field leakage on the activation barrier of the translocation events. When field leakage is involved, the driving field through the pore tip is reduced from $E_{0}$ to $E_{\text {water }}^{\text {normal }}$. Thus, there is a force $q E_{\text {water }}^{\text {normal }}$ which pulls the molecule into the pore, and one $q E_{\text {leak }}$ that opposes it (see Fig. 2c). Assuming field leakage to be uniform for all base pairs outside the pore (and not to affect the bases inside it), these forces may be integrated from $z=0$ (all bps outside) to $z=L$ (all bps inside), leading to an estimate of the barrier reduction (see Supplementary Note 2)

$$
W=L q\left[E_{\text {water }}^{\text {normal }}-E_{\text {leak }} / 2\right] \text {, }
$$

with $L$ taken as the total length of the molecule (comparable to the sensing region of our pores). Decreasing the thickness $l$ of the $\mathrm{Al}_{2} \mathrm{O}_{3}$

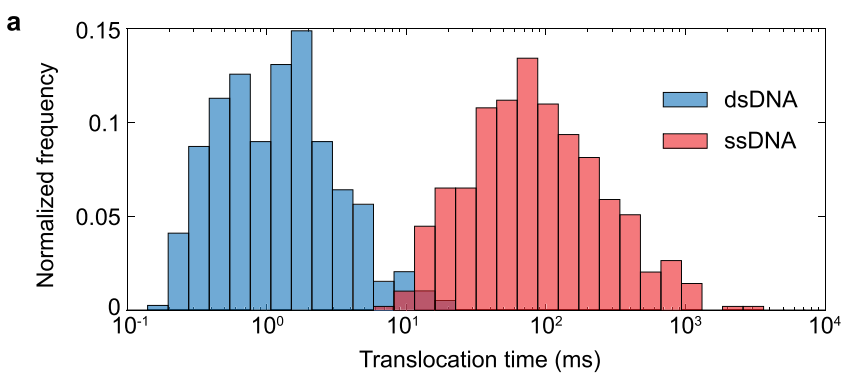

b

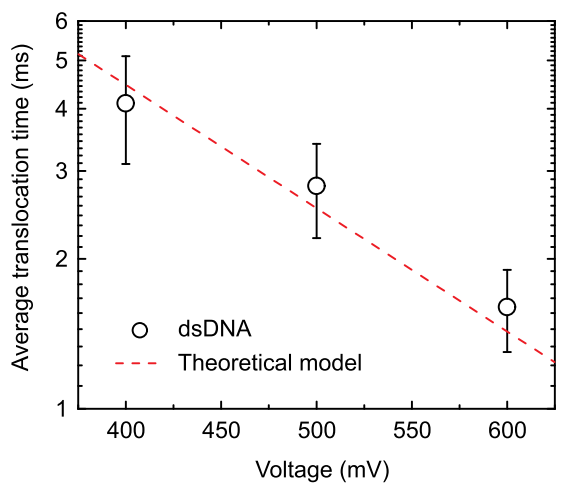

c

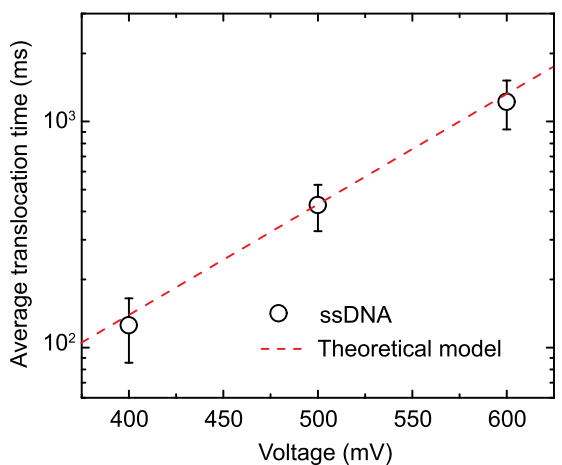

Fig. 6 Comparison between dsDNA and ssDNA experimental

translocation times. a Normalized histogram of translocation times for 22 nt ssDNA ( $n=390$ events) and 22 bp dsDNA ( $n=492$ events). ssDNA translocates much slower than dsDNA under the effect of electric field leakage (for a nanopore with a diameter of $10 \mathrm{~nm}$, half cone angle $\sim \pm 2^{\circ}$ coated with $3 \mathrm{~nm} \mathrm{Al} \mathrm{O}_{3}$ film under an applied voltage of $500 \mathrm{mV}$ ). These signature electrical signals allow discrimination (>97\%) between ssDNA and dsDNA duplex translocation events (see Supplementary Fig. 6). b Average dsDNA translocation time dependence as a function of the applied voltage $(n=3)$. The line represents the fit of the data to the model ( $\tau=\tau_{0} e^{-W / k_{B} T}$, where $W$ is given by Eq. (3) with $\tau_{0}=45$ ms for a dsDNA with a charge per bp of $0.5 \mathrm{e}$ and a length per bp of $0.34 \mathrm{~nm}^{70}$ ). c Average ssDNA translocation time as a function of the applied voltage $(n=3)$. The line represents the fit of the data to the model $\left(\tau=\tau_{0} e^{-\left(W+W^{*}\right) / k_{B} T}\right.$, where $W$ and $W^{\star}$ are given by Eqs. (3) and (4), respectively, with $\tau_{0}=1.595 \mathrm{~ms}$, for a charge per nt of 0.1 e and a length per nt of $0.64 \mathrm{~nm}$ ). Error bars in the figures represent the standard deviation between independent experiments.

leads to higher field leakage and lower fields inside the pore, reducing $W$ and leading to slower translocation events. For sufficiently long nanopores and small values of $l$, we may consider $E_{\text {leak }}=E_{\text {water }}^{\text {normal }}\left(\varepsilon_{\text {film }} / \varepsilon_{\text {water }}\right)$, and Eq. (3) can be simplified to $W=$ $L q E_{\text {leak }}\left[\varepsilon_{\text {water }} / \varepsilon_{\text {film }}-1 / 2\right]$. Estimating $E_{\text {leak }}$ from Eq. (1), we find excellent quantitative agreement to translocation time data for dsDNA if we reduce the relative permittivity of water $\varepsilon_{\text {water }}$ from 80 
to 6 (see Fig. 6b). This adjustment is reasonable, as the dielectric constant of surficial water layer of 2-3 molecules thick is known to be significantly smaller than that of bulk water (the literature value is $2-20^{60,61}$ ) because the rotational freedom of water dipoles decreases for the immobile layers near the surface. Thus, the normal field leakage $E_{\text {leak }}$ near the pore edge surface becomes higher than normally expected because of the weak screening effect of the immobile water layers. A water permittivity comparable to the dielectric film would indeed produce a leakage field that is comparable to the applied field, according to the thin-film limit of Eq. (1).

As $\varepsilon_{\text {water }} / \varepsilon_{\text {film }}>1 / 2$, our previous derivation suggests that translocation time should still decrease exponentially with increasing voltage, even though the field leakage has increased the barrier as it reduces the pulling force. This is true for dsDNA. However, we found a curious opposite trend for the $22 \mathrm{nt}$ ssDNA (see Fig. 6c), whose translocation time increased exponentially with the applied voltage. This suggests that other than adjusting for the total number of charges q, Eq. (3) needs to be modified for ssDNA. ssDNA molecules uncoil near hydrophobic surfaces, maximizing their hydrophobic interactions by means of increasing the contacts between the surface and their exposed aromatic rings ${ }^{62}$. In dsDNA molecules, this mechanism would require the breakage of the hydrogen bonds that stabilize the complementary base-pairs ${ }^{63-65}$. Therefore, hydrophobic interactions between dsDNA molecules and charged surfaces are limited to the two end base pairs, which have been suggested to present rapid (pico-seconds) opening and closing dynamics $^{66}$. To cater to hydrophobic interactions present in ssDNA (and absent in their double stranded counterparts), a voltagedependent term $W^{*}$ will be added to Eq. (3), which will model the van der Waals attraction of the hydrophobic rings of ssDNA towards the surface. We assume this term to be proportional to the field and a positive contribution to the barrier results,

$$
W^{*}=-k L q E_{\text {leak }}
$$

as field leakage brings the molecule into closer proximity to the membrane, stretching the molecule and thus increasing its affinity to the surface. Excellent agreement of our theory with experimental data is found by considering $k \sim 0.9$ (see Fig. 6c), suggesting hydrophobic interactions amplifies electrostatic pinning of ssDNA molecules to the pore surface, to the extent that physical adsorption occurs. The additional term in Eq. (4) only applies to ssDNA, leading to $W+W^{*}=L q E_{\text {leak }}\left(\varepsilon_{\text {water }} / \varepsilon_{\text {film }}-1 / 2-k\right)<0$ and a translocation time $\tau=\tau_{0} e^{-\left(W+W^{*}\right) / k_{\mathrm{B}} T}$ which increases with the applied voltage.

Our theoretical model has shown to work for short 22 nt DNA. However, this theory assumes all nucleotides in contact with the outer membrane feel the same leakage field. While this may be a good assumption for short molecules, it breaks down when $L \gg l$ because the leakage field is only localized at the pore orifice around the alumina layer and larger molecules will also feel more electrophoretic force that drives them into the pore. The pinning effect of the leakage field will hence be overwhelmed for long molecules. As a result, we expect faster translocation velocity for longer DNAs, which is consistent with our measurements shown in Supplementary Fig. 7. In other words, the field leakage effect is more effective for slowing down shorter ssDNA translocation. The normalized DNA velocity (nt $\mathrm{ms}^{-1}$ ) is observed to scale linearly with the DNA length since longer DNA carries more charge and thus experiences greater electrophoretic driving force for translocation. Nevertheless, the normalized translocation velocity for a $200 \mathrm{nt}$ ssDNA translocating through the $\mathrm{Al}_{2} \mathrm{O}_{3}$-PET nanopore is around $50 \mathrm{nt} \mathrm{ms}^{-1}$, which is still much slower than the typical velocity $\left(10^{3}-10^{5} \mathrm{nt} \mathrm{ms}^{-1}\right)$ in other solid-state nanopores (Supplementary Fig. 7).
This adsorption of ssDNA molecules to the pore surface is further confirmed by analyzing the current change associated to the translocation events. Due to their smaller cross-section area, single stranded molecules block less current than their double stranded counterparts, leading to lower current drops at high salt concentrations (such as the ones used in our experiments) ${ }^{67,68}$ (see Fig. 7a). However, we observe comparable, if not higher,

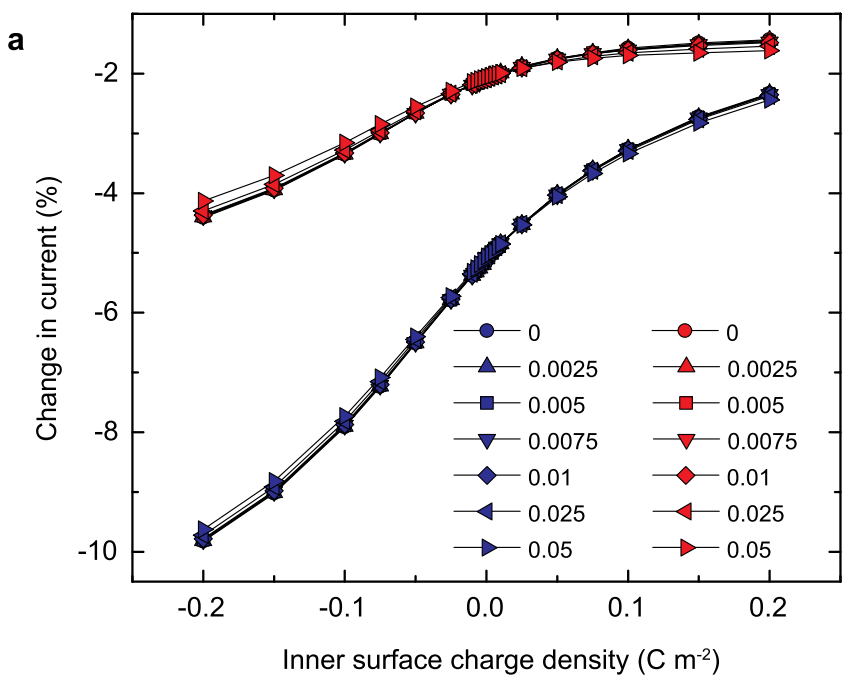

b

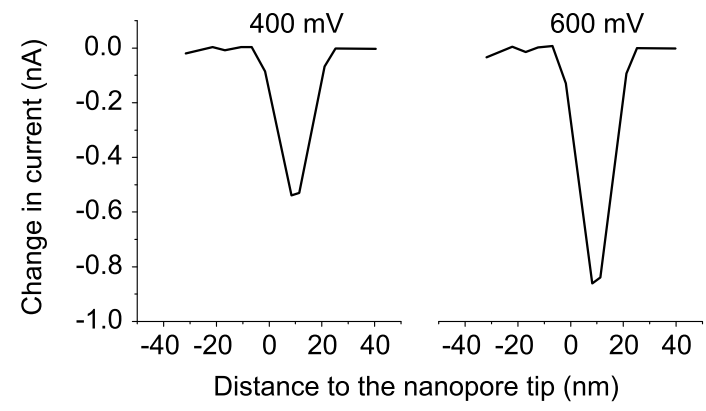

c

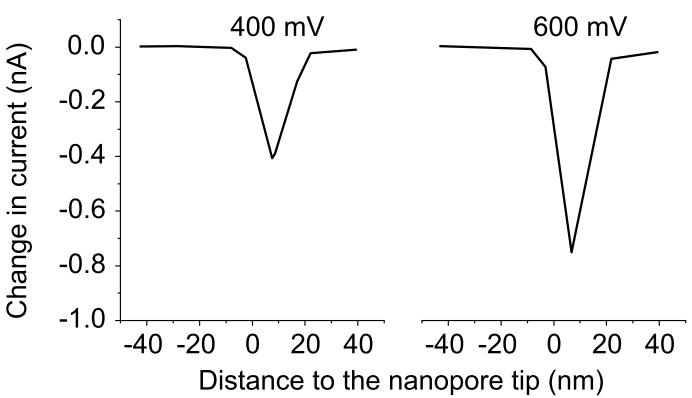

Fig. 7 Finite-element-method simulation of the resistive signals. a FEM simulated normalized change in current for a dsDNA molecule (blue) and a ssDNA molecule (red) translocating through the axis of a nanopore in function of the inner (horizontal axis) and outer (symbol shape) surface charge density at $500 \mathrm{mV}$. Note that dsDNA molecules always lead to higher current drops than their single stranded counterparts, as they have higher cross-section areas. b FEM simulated current drops for single stranded molecules translocating through the walls of the nanopore at 400 and $600 \mathrm{mV}$. c FEM simulated current drops for double stranded molecules translocating through the axis of the nanopore at 400 and $600 \mathrm{mV}$. Note that ssDNA molecules translocating through the pore walls present comparable resistive signals with their double stranded counterparts translocating through the bulk of the nanopore, in agreement with Figs. 3 and 5. Simulation details are presented in Supplementary Note 3. 

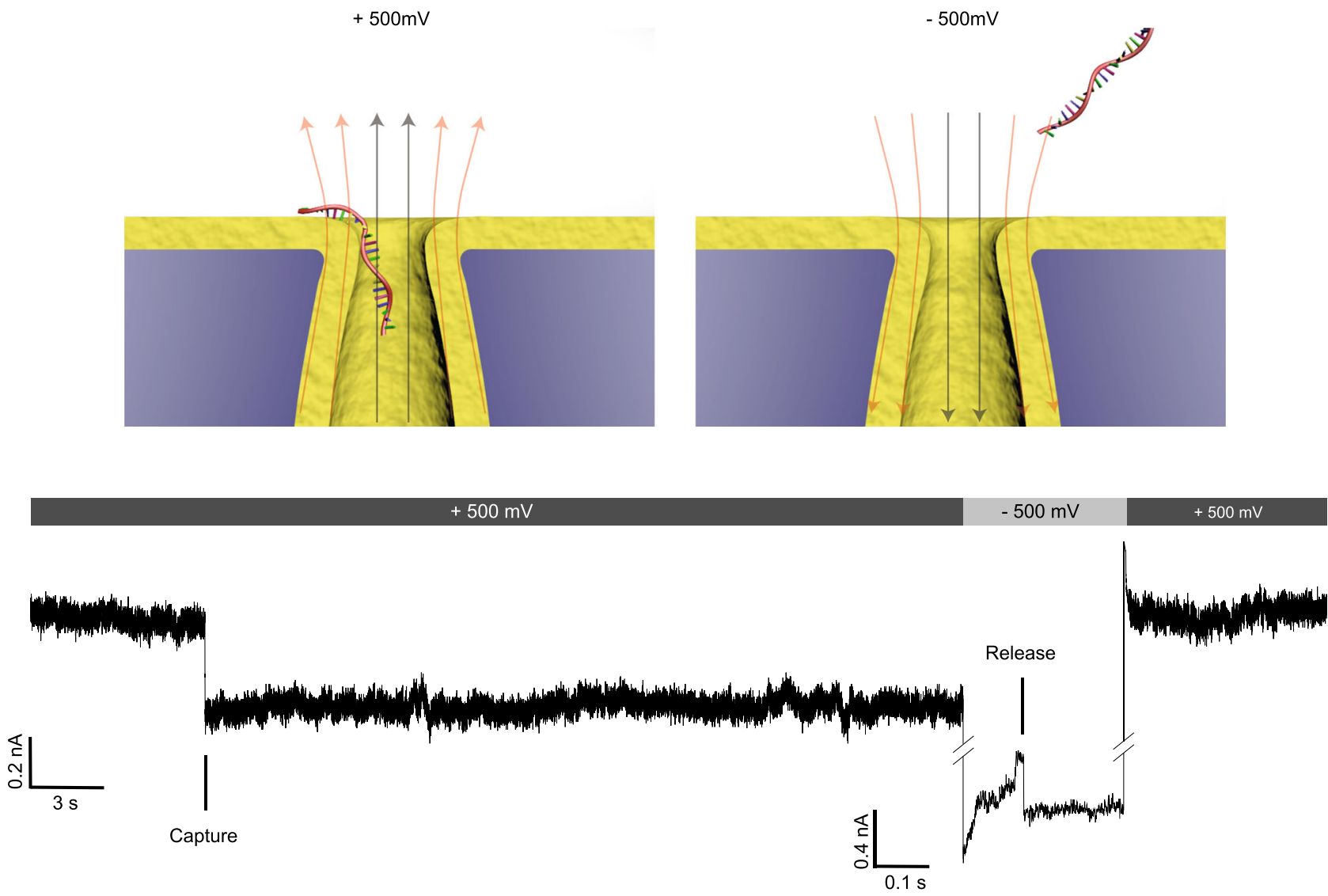

Fig. 8 Reversibility of the molecular pinning. Electrical recording of $22 \mathrm{nt}$ ssDNA pinning at the pore edge of a bullet-shaped $\mathrm{Al}_{2} \mathrm{O}_{3}$-coated (thickness, $3 \mathrm{~nm}$ ) nanopores (diameter, $10 \mathrm{~nm}$ ) with a half cone angles of $20 \pm 3^{\circ}$ under applied voltage of $+500 \mathrm{mV}$ (energy barrier, $12.7 \mathrm{kT}$ ) and ssDNA escaping from the nanopore after the electrostatic trapping effect is switched off by reversing the polarity of applied voltage to $-500 \mathrm{mV}$.

current drops for our single stranded molecule experiments (see Figs. 3 and 5), which can only result from significant modulation of the ionic conductivity due to surface effects ${ }^{62}$. In conical and bullet-shaped nanopores, the sensing area is localized near the tip, where the field is focused ${ }^{51}$ and the surface charge of the thin alumina coating controls the ionic conductance in this key region. Adsorption of molecules near this region will then change the effective surface charge density of the pore walls (lowering it in our case, as $\mathrm{Al}_{2} \mathrm{O}_{3}$ is positively charged), depleting their counterions and leading to lower current. This would then lead to much higher current drops than for molecules translocating far from the pore walls ${ }^{62}$ (see Fig. 7b). We have reproduced the resistive current pulses of $22 \mathrm{nt}$ ssDNA and $22 \mathrm{bp}$ dsDNA by FEM simulation (Fig. 7b, c), with comparable amplitudes for both due to adsorption of ssDNA.

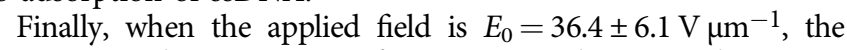
average translocation time of ssDNA can last several minutes (Fig. 4a). Even under such strong normal leakage field, this electrostatic trapping effect is completely reversible. This is illustrated in Fig. 8, where the current recovered to the base level after a negative voltage was applied. Once the direction of applied voltage and normal leakage field is reversed, the electrostatic trapping effect is switched off, and ssDNA can escape from the nanopore (see also Supplementary Fig. 8). This reversible electrostatic trapping effect reduces the possibility of permanent nanopore blocking by translocating molecules, a common issue for nanopore sensors $^{69}$. It is worth noting that the observed long translocation time does not imply that every base translocates through the nanopore at the same slow velocity. The translocation kinetics through the $\mathrm{Al}_{2} \mathrm{O}_{3}$-PET nanopores resemble a "stick-slip" motion in which the DNA rapidly translocates as soon as the applied force overcomes the energy barrier. A stick-slip translocation mechanism may give long dwell times for only a single segment along a DNA, which is not helpful for resolving the other segments and unfavorable in nanopore-sequencing technology. On the other hand, the high translocation speed of DNA is probably not the biggest problem for sequencing with solid-state nanopores. The main problem is that the ionic current signal in solid-state nanopores does not provide the resolution for base determination.

\section{Discussion}

We have designed dielectric film coated solid-state nanopores in insulating polymer membranes, with an enhanced leakage field at the pore edge that can delay the translocation of ssDNA molecules by 5 orders of magnitude, thus providing a practical method to achieve up to 5 orders of temporal resolution enhancement for sensing applications. This large range of translocation times is due to an activated transport mechanism into the pore endowed by the pinning field, which can lead to actual adsorption, with an exponential dependence on the applied field and a barrier height that is sensitive to the affinity of the molecule to the surface. The prolonged molecular-pinning time allows short nucleic acids to produce observable and distinct resistive signals and yet does not clog the nanopores or interfere with the resistive signal current. The reported enhanced sensitivity and selectivity would be useful for multiplex profiling of target microRNAs after hybridizing them with designed bar-coded oligos with dangling tails of different signature translocation times within the large range 
reported here. That the pinned ssDNA actually absorbs onto the edge suggests specific sequences or protein attachments can increase the library volume. Further studies of the interactions between DNA and normal field leakage in the context of voltagedriven DNA translocation may allow DNA translocate through the nanopore base-by-base, enabling a more controlled transport through nanopores equipped with transverse electrodes and allowing high-resolution sequencing or DNA/protein interaction analyses.

\section{Methods}

Fabrication of $\mathbf{A l}_{2} \mathbf{O}_{3}$-coated polymeric nanopore. The $12 \mu \mathrm{m}$ thick PET foils were irradiated with single swift heavy ions (Au) with energy of $11.4 \mathrm{MeV}$ per nucleon at the GSI in Darmstadt, Germany. An irradiated foil was subsequently etched at room temperature $(295 \mathrm{~K})$ by an asymmetric etching method, where the foil was mounted between two isolated containers that contained an etchant solution of $2.5 \mathrm{M} \mathrm{NaOH}$ in $1: 1 \mathrm{MeOH} / \mathrm{H}_{2} \mathrm{O}$, a stopping solution of $1 \mathrm{M}$ $\mathrm{HCOOH}$, and $1 \mathrm{M} \mathrm{KCl}$ aqueous solution, respectively. The etching process started from one side of the PET foil, but was immediately stopped when etched through, and as a result, a single trumpet-like nanopore was formed on each irradiated PET foil. Bullet-shaped nanopores with different half cone angles were fabricated by tuning etching times after breakthrough (see Supplementary Fig. 3). A secondary symmetric etching process $(2 \mathrm{M} \mathrm{NaOH})$ was applied to enlarge the tip size. In all cases, the radius of the base was around $500 \pm 80 \mathrm{~nm}$, as determined by electron microscopy. The final tip radius was determined by a conductance measurement. Thermal ALD $\mathrm{Al}_{2} \mathrm{O}_{3}$ films of 3 or $10 \mathrm{~nm}$ were grown in a commercial (Cambridge Nanotech, Savannah S100) ALD reactor using trimethylaluminium (TMA) and de-ionized (DI) water as precursors. Due to the self-limiting nature of the ALD surface chemistry reactions, the film thickness was precisely controlled by setting a certain number of the ALD cycles. An ALD growth cycle of $\mathrm{Al}_{2} \mathrm{O}_{3}$ deposition consists of the following steps and parameters: TMA pulse $0.02 \mathrm{~s}, \mathrm{~N}_{2}$ purge $15 \mathrm{~s}, \mathrm{H}_{2} \mathrm{O}$ pulse $0.02 \mathrm{~s}, \mathrm{~N}_{2}$ purge $20 \mathrm{~s}$. A low deposition temperature of $110^{\circ} \mathrm{C}$ was chosen to prevent thermal damage to the polymer PET.

Experiments of DNA transport. A PET foil with a single $\mathrm{Al}_{2} \mathrm{O}_{3}$-coated polymeric nanopore was mounted between two isolated channels that were both filled with buffered $1 \mathrm{M} \mathrm{KCl}$ aqueous solution $(0.01 \times \mathrm{PBS}, \mathrm{pH}=7.4)$. A patch clamp amplifier (Axopatch 200B, Molecular Devices Inc.) with $\mathrm{Ag} / \mathrm{AgCl}$ electrodes was used to measure the current trace and the current-voltage response across the nanopore. The polarity of the applied voltage was referenced to the tip side electrode. The current data were collected at 50 or $100 \mathrm{kHz}$ with a low-pass Bessel filter of $10 \mathrm{kHz}$. For the DNA transport experiment, the buffered $10 \mathrm{pM}$ $22 \mathrm{nt}$ ssDNA and $22 \mathrm{bp}$ dsDNA (Integrated DNA Technologies) solution (in $1 \mathrm{M}$ $\mathrm{KCl}, 0.01 \times \mathrm{PBS}, \mathrm{pH}=7.4$ ) was always freshly made prior to each experiment and was injected to the tip side of the nanopore; $22 \mathrm{bp}$ dsDNA was obtained by hybridizing two complementary oligos and then purified by gel electrophoresis. Unless otherwise specified, a positive voltage of $500 \mathrm{mV}$ was used in the transport experiment to drive the negatively charged molecules through the nanopore from tip to base.

Finite-element-method simulations. All FEM simulations were performed with the commercial code COMSOL. Simulation details of the field leakage are presented in Supplementary Note 1. Simulations of the translocation events were performed following a methodology previously published ${ }^{62}$. The $2 \mathrm{D}$ simulations were performed for the axisymmetric systems (bulk translocations) while 3D simulations were performed for the non-symmetric systems (adsorption). For each simulation, an initial mesh heavily refined on narrow regions, near the translocating agents, and near charged surfaces was considered. This mesh was refined through 5 mesh adaptation steps and convergence of the current was assessed during these adaptations (see Supplementary Note 3).

\section{Data availability}

Data supporting the findings of this paper are available from the corresponding authors upon reasonable request. The source data underlying Figs. 1b-d, 2, 3, 4a, c, 5, 6, 7, 8 and Supplementary Figs. 2, 3, 4, 5, 6, 7, 8, 12, 13, and 14 are provided as a Source Data file (https://doi.org/10.6084/m9.figshare.13238687.v1).

\section{Code availability}

Code for data analysis is available from the corresponding author upon reasonable request.

Received: 17 July 2020; Accepted: 23 November 2020; Published online: 08 January 2021

\section{References}

1. Venkatesan, B. M. \& Bashir, R. Nanopore sensors for nucleic acid analysis. Nat. Nanotechnol. 6, 615-624 (2011).

2. Wanunu, M. Nanopores: a journey towards DNA sequencing. Phys. Life Rev. 9, 125-158 (2012)

3. Deamer, D., Akeson, M. \& Branton, D. Three decades of nanopore sequencing. Nat. Biotechnol. 34, 518-524 (2016).

4. Yusko, E. C. et al. Controlling protein translocation through nanopores with bio-inspired fluid walls. Nat. Nanotechnol. 6, 253-260 (2011).

5. Kennedy, E., Dong, Z., Tennant, C. \& Timp, G. Reading the primary structure of a protein with $0.07 \mathrm{~nm}^{3}$ resolution using a subnanometre-diameter pore. Nat. Nanotechnol. 11, 968-976 (2016).

6. Smeets, R. M. M., Kowalczyk, S. W., Hall, A. R., Dekker, N. H. \& Dekker, C. Translocation of RecA-coated double-stranded DNA through solid-state nanopores. Nano Lett. 9, 3089-3095 (2009).

7. Dekker, C. Solid-state nanopores. Nat. Nanotechnol. 2, 209-215 (2007).

8. Branton, D. et al. The potential and challenges of nanopore sequencing. Nat. Biotechnol. 26, 1146-1153 (2008).

9. Di Ventra, M. \& Taniguchi, M. Decoding DNA, RNA and peptides with quantum tunnelling. Nat. Nanotechnol. 11, 117-126 (2016).

10. Fologea, D., Uplinger, J., Thomas, B., McNabb, D. S. \& Li, J. Slowing DNA translocation in a solid-state nanopore. Nano Lett. 5, 1734-1737 (2005).

11. Meller, A., Nivon, L. \& Branton, D. Voltage-driven DNA translocations through a nanopore. Phys. Rev. Lett. 86, 3435-3438 (2001).

12. Muthukumar, M. Mechanism of DNA transport through pores. Annu. Rev. Biophys. Biomolecular Struct. 36, 435-450 (2007).

13. Plesa, C. et al. Fast translocation of proteins through solid state nanopores. Nano Lett. 13, 658-663 (2013).

14. Rosenstein, J. K., Wanunu, M., Merchant, C. A., Drndic, M. \& Shepard, K. L. Integrated nanopore sensing platform with sub-microsecond temporal resolution. Nat. Methods 9, 487-492 (2012).

15. Venta, K. et al. Differentiation of short, single-stranded DNA homopolymers in solid-state nanopores. ACS Nano 7, 4629-4636 (2013).

16. Smeets, R. M. M., Keyser, U. F., Dekker, N. H. \& Dekker, C. Noise in solidstate nanopores. Proc. Natl. Acad. Sci. USA 105, 417-421 (2008).

17. Wanunu, M. et al. Rapid electronic detection of probe-specific microRNAs using thin nanopore sensors. Nat. Nanotechnol. 5, 807-814 (2010).

18. Wang, Y., Zheng, D., Tan, Q., Wang, M. \& Gu, L.-Q. Nanopore-based detection of circulating microRNAs in lung cancer patients. Nat. Nanotechnol. 6, 668-674 (2011)

19. Yokota, K., Tsutsui, M. \& Taniguchi, M. Electrode-embedded nanopores for label-free single-molecule sequencing by electric currents. RSC Adv. 4, 15886-15899 (2014)

20. Yuan, Z., Liu, Y., Dai, M., Yi, X. \& Wang, C. Controlling DNA translocation through solid-state nanopores. Nanoscale Res. Lett. 15, 80 (2020).

21. Kowalczyk, S. W., Wells, D. B., Aksimentiev, A. \& Dekker, C. Slowing down DNA translocation through a nanopore in lithium chloride. Nano Lett. 12, 1038-1044 (2012)

22. Yeh, L.-H., Zhang, M., Joo, S. W. \& Qian, S. Slowing down DNA translocation through a nanopore by lowering fluid temperature. Electrophoresis 33, 3458-3465 (2012).

23. Luan, B. et al. Base-by-base ratcheting of single stranded DNA through a solid-state nanopore. Phys. Rev. Lett. 104, 238103 (2010).

24. Verschueren, D. V. et al. Label-free optical detection of DNA translocations through plasmonic nanopores. ACS Nano 13, 61-70 (2019).

25. Hall, A. R. et al. Hybrid pore formation by directed insertion of alpha hemolysin into solid-state nanopores. Nat. Nanotechnol. 5, 874-877 (2010).

26. Cherf, G. M. et al. Automated forward and reverse ratcheting of DNA in a nanopore at 5-Å precision. Nat. Biotechnol. 30, 344-348 (2012).

27. Manrao, E. et al. Reading DNA at a single-nucleotide resolution with a mutant MspA nanopore and phi29 DNA polymerase. Nat. Biotechnol. 30, 349-353 (2012).

28. Nam, S.-W., Rooks, M. J., Kim, K.-B. \& Rossnagel, S. M. Ionic field effect transistors with sub-10 nm multiple nanopores. Nano Lett. 9, 2044-2048 (2009).

29. Banerjee, S. et al. Slowing DNA transport using graphene-DNA interactions. Adv. Funct. Mater. 25, 936-946 (2015).

30. Larkin, J. et al. Slow DNA transport through nanopores in hafnium oxide membranes. ACS Nano 7, 10121-10128 (2013).

31. Jiang, Z. \& Stein, D. Charge regulation in nanopore ionic field-effect transistors. Phys. Rev. E 83, 031203 (2011).

32. Venkatesan, B. M. et al. Stacked graphene-Al2O3 nanopore sensors for sensitive detection of DNA and DNA-protein complexes. ACS Nano 6, 441-450 (2012).

33. Di Fiori, N. et al. Optoelectronic control of surface charge and translocation dynamics in solid-state nanopores. Nat. Nanotechnol. 8, 946-951 (2013).

34. Miles, B. N. et al. Single molecule sensing with solid-state nanopores: novel materials, methods, and applications. Chem. Soc. Rev. 42, 15-28 (2012). 
35. Venkatesan, B. M., Shah, A. B., Zuo, J.-M. \& Bashir, R. DNA sensing using nanocrystalline surface-enhanced $\mathrm{Al} 2 \mathrm{O} 3$ nanopore sensors. Adv. Funct. Mater. 20, 1266-1275 (2010).

36. Stein, D. et al. Electrokinetic concentration of DNA polymers in nanofluidic channels. Nano Lett. 10, 765-772 (2010).

37. Murarka, S. P. Silicon dioxide, nitride, and oxynitride. In Encyclopedia of Materials: Science and Technology (eds Buschow, K. H. J. et al.) 1-14 (Elsevier, 2003). https://doi.org/10.1016/B0-08-043152-6/01895-7.

38. Pedroli, F. et al. Reducing leakage current and dielectric losses of electroactive polymers through electro-annealing for high-voltage actuation. RSC Adv. 9, 12823-12835 (2019).

39. Schroeder, H. \& Schmitz, S. Thickness dependence of leakage currents in high-permittivity thin films. Appl. Phys. Lett. 83, 4381-4383 (2003).

40. Sarma, K. C. D. \& Sharma, S. A method for reduction of off state leakage current in symmetric DG JLT. Eng. Res. Express 1, 015034 (2019).

41. Atanassova, E. \& Dimitrova, T. Thin Ta2O5 Layers on $\mathrm{Si}$ as an alternative to $\mathrm{SiO}_{2}$ for high-density DRAM applications. In Handbook of Surfaces and Interfaces of Materials (ed. Nalwa, H.S.) Ch. 9, 439-479 (Academic Press, 2001). https://doi.org/10.1016/B978-012513910-6/50055-4.

42. Lin, H. C., Ye, P. D. \& Wilk, G. D. Leakage current and breakdown electricfield studies on ultrathin atomic-layer-deposited $\mathrm{Al}_{2} \mathrm{O}_{3}$ on GaAs. Appl. Phys. Lett. 87, 182904 (2005).

43. Thamida, S. K. \& Chang, H.-C. Nonlinear electrokinetic ejection and entrainment due to polarization at nearly insulated wedges. Phys. Fluids 14, 4315-4328 (2002).

44. Eckstein, Y., Yossifon, G., Seifert, A. \& Miloh, T. Nonlinear electrokinetic phenomena around nearly insulated sharp tips in microflows. J. Colloid Interface Sci. 338, 243-249 (2009).

45. Gramotnev, D. K. \& Bozhevolnyi, S. I. Nanofocusing of electromagnetic radiation. Nat. Photonics 8, 13-22 (2014)

46. Deygout, J. Multiple knife-edge diffraction of microwaves. IEEE Trans. Antennas Propag. 14, 480-489 (1966).

47. Collins, R. T., Sambath, K., Harris, M. T. \& Basaran, O. A. Universal scaling laws for the disintegration of electrified drops. Proc. Natl. Acad. Sci. USA 110, 4905-4910 (2013).

48. Tabard-Cossa, V., Trivedi, D., Wiggin, M., Jetha, N. N. \& Marziali, A. Noise analysis and reduction in solid-state nanopores. Nanotechnology 18, 305505 (2007).

49. Lee, M.-H. et al. A low-noise solid-state nanopore platform based on a highly insulating substrate. Sci. Rep. 4, 7448 (2014).

50. Apel, P. Y., Korchev, Yu. E., Siwy, Z., Spohr, R. \& Yoshida, M. Diode-like single-ion track membrane prepared by electro-stopping. Nucl. Instrum. Methods Phys. Res. B 184, 337-346 (2001).

51. Wang, C. et al. Atomic layer deposition modified track-etched conical nanochannels for protein sensing. Anal. Chem. 87, 8227-8233 (2015).

52. Py, A. et al. Shedding light on the mechanism of asymmetric track etching: an interplay between latent track structure, etchant diffusion and osmotic flow. Phys. Chem. Chem. Phys. 18, 25421-25433 (2016).

53. $\mathrm{Wu}, \mathrm{L}$. et al. Electrically facilitated translocation of protein through solid nanopore. Nanoscale Res. Lett. 9, 140 (2014).

54. Liu, Q. et al. Voltage-driven translocation of DNA through a high throughput conical solid-state nanopore. PLoS ONE 7, e46014 (2012).

55. Zhang, J. \& Shklovskii, B. I. Effective charge and free energy of DNA inside an ion channel. Phys. Rev. E 75, 021906 (2007).

56. Wanunu, M., Sutin, J., McNally, B., Chow, A. \& Meller, A. DNA translocation governed by interactions with solid-state nanopores. Biophys. J. 95, 4716-4725 (2008).

57. Ghosal, S., Sherwood, J. D. \& Chang, H.-C. Solid-state nanopore hydrodynamics and transport. Biomicrofluidics 13, 011301 (2019).

58. Chang, H. \& Yeo, L. Electrokinetically Driven Microfluidics and Nanofluidics (Cambridge University Press, 2010).

59. Ghosal, S. Electrophoresis of a polyelectrolyte through a nanopore. Phys. Rev. E 74, 041901 (2006).

60. Fumagalli, L. et al. Anomalously low dielectric constant of confined water. Science 360, 1339-1342 (2018).

61. Cherepanov, D. A., Feniouk, B. A., Junge, W. \& Mulkidjanian, A. Y. Low dielectric permittivity of water at the membrane interface: effect on the energy coupling mechanism in biological membranes. Biophys. J. 85, 1307-1316 (2003).

62. Sensale, S., Wang, C. \& Chang, H.-C. Resistive amplitude fingerprints during translocation of linear molecules through charged solid-state nanopores. J. Chem. Phys. 153, 085102 (2020).
63. Kwon, K. D., Vadillo-Rodriguez, V., Logan, B. E. \& Kubicki, J. D. Interactions of biopolymers with silica surfaces: force measurements and electronic structure calculation studies. Geochim. Cosmochim. Acta 70, 3803-3819 (2006).

64. Isailovic, S., Li, H.-W. \& Yeung, E. S. Adsorption of single DNA molecules at the water/fused-silica interface. J. Chromatogr. A 1150, 259-266 (2007).

65. Shi, B., Shin, Y. K., Hassanali, A. A. \& Singer, S. J. DNA binding to the silica surface. J. Phys. Chem. B 119, 11030-11040 (2015).

66. Andreatta, D. et al. Ultrafast dynamics in DNA: "Fraying" at the end of the helix. J. Am. Chem. Soc. 128, 6885-6892 (2006).

67. Smeets, R. M. M. et al. Salt dependence of ion transport and DNA translocation through solid-state nanopores. Nano Lett. 6, 89-95 (2006).

68. Skinner, G. M., van den Hout, M., Broekmans, O., Dekker, C. \& Dekker, N. H. Distinguishing single- and double-stranded nucleic acid molecules using solidstate nanopores. Nano Lett. 9, 2953-2960 (2009).

69. Agah, S., Zheng, M., Pasquali, M. \& Kolomeisky, A. B. DNA sequencing by nanopores: advances and challenges. J. Phys. D 49, 413001 (2016).

70. Sensale, S., Peng, Z. \& Chang, H.-C. Biphasic signals during nanopore translocation of DNA and nanoparticles due to strong ion cloud deformation. Nanoscale 11, 22772-22779 (2019).

\section{Acknowledgements}

This work has been supported by the National Institutes of Health (IMAT Grant No. R21CA206904) and the NIH Common Fund, through the Office of Strategic Coordination/Office of the NIH Director, 1UG3CA241684-01. The authors acknowledge that ZP was supported by a China Scholarship Council fellowship. We are grateful to Christina Trautmann, Yugang Wang, and Jianming Xue for providing the irradiated PET membrane samples.

\section{Author contributions}

H.C.C. and C.W. conceived the project and designed the experiments. C.W. performed the experimental studies. S. Senapati performed the DNA purification. H.C.C., S. Sensale, C.W., and Z.P. performed the theoretical analysis. C.W., S. Sensale, and H.C.C. wrote the paper. H.C.C. supervised the work. All authors discussed the results and commented on the paper.

\section{Competing interests}

The authors declare no competing interests.

\section{Additional information}

Supplementary information is available for this paper at https://doi.org/10.1038/s41467 020-20409-4.

Correspondence and requests for materials should be addressed to H.-C.C.

Peer review information Nature Communications thanks Jiali Li and Derek Stein for their contribution to the peer review of this work.

Reprints and permission information is available at http://www.nature.com/reprints

Publisher's note Springer Nature remains neutral with regard to jurisdictional claims in published maps and institutional affiliations.

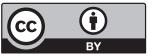

Open Access This article is licensed under a Creative Commons Attribution 4.0 International License, which permits use, sharing, adaptation, distribution and reproduction in any medium or format, as long as you give appropriate credit to the original author(s) and the source, provide a link to the Creative Commons license, and indicate if changes were made. The images or other third party material in this article are included in the article's Creative Commons license, unless indicated otherwise in a credit line to the material. If material is not included in the article's Creative Commons license and your intended use is not permitted by statutory regulation or exceeds the permitted use, you will need to obtain permission directly from the copyright holder. To view a copy of this license, visit http://creativecommons.org/ licenses/by/4.0/.

(C) The Author(s) 2021 